\title{
Producción científica sobre ansiedad bibliotecaria: un análisis bibliométrico y cienciométrico desde Scopus
}

\author{
Pier Suclupe-Navarro*, Cesar H. Limaymanta*,**, Néstor Holmes Ramírez***, Héctor Guillén* \\ * Universidad Nacional Mayor de San Marcos. Perú \\ Correo-e: pier.suclupe@unmsm.edu.pe | ORCID iD: https://orcid.org/0000-0001-7232-2103 \\ Correo-e: climaymantaa@unmsm.edu.pe | ORCID-iD: http://orcid.org/0000-0002-8797-4275 \\ Correo-e: hector.guillen1@unmsm.edu.pe | ORCID iD: https://orcid.org/0000-0002-2644-8167 \\ **Universidad Peruana de Ciencias Aplicadas (UPC). Perú \\ Correo-e: pcmaclim@upc.edu.pe | ORCID-iD: http://orcid.org/0000-0002-8797-4275 \\ ***Biblioteca de la Universidad de Montemorelos. México \\ Correo-e: nestor@um.edu.mx | ORCID iD: https://orcid.org/0000-0001-5597-4513
}

Recibido: 29-01-20; 2a versión: 23-04-20; Aceptado: 27-04-20; Publicado: 29-04-2021

Cómo citar este artículo/Citation: Suclupe-Navarro, P.; Limaymanta, C.H.; Ramírez, N.H.; Guillén, H. (2021) Producción científica sobre ansiedad bibliotecaria: un análisis bibliométrico y cienciométrico desde Scopus. Revista Española de Documentación Científica, 44 (2), e291. https://doi.org/10.3989/redc.2021.2.1753

Resumen: La ansiedad bibliotecaria $(A B)$ es el estado de incertidumbre que experimentan algunos usuarios cuando acuden a la biblioteca, cuyo estudio comenzó en los años 80, pero que carece de un análisis métrico actualizado. El objetivo de este estudio fue hacer un análisis bibliométrico y cienciométrico de los artículos sobre $A B$ indizados en la base de datos Scopus entre 1989 y 2018. Se analizaron 118 publicaciones según indicadores de producción, colaboración, impacto y mapas bibliométricos. Se encontró que las publicaciones sobre AB han aumentado progresivamente, que la colaboración de autores fue baja, que una revista influyente en el área fue Library Review, y que los autores con más impacto fueron Onwuegbuzie y Jiao. Asimismo, destacaron palabras clave sobre la formación del usuario y cuatro frentes de investigación: instrumentos de medición, resultados, marcos teóricos y tipos de unidades de análisis. Dichos resultados sugieren que la $A B$ es un tema fructífero de investigación.

Palabras clave: ansiedad bibliotecaria; Bibliometría; Cienciometría; usuarios de información; Scopus; VoSviewer; emparejamiento bibliográfico; cocitación.

\section{Scientific p roduction o n I ibrary a nxiety: a b ibliometric a nd s cientometric a nalysis from Scopus}

Abstract: Library anxiety (LA) is the state of uncertainty experienced by some users when they come to the library, the study of which began in the 1980 s but lacks updated metric analysis. The objective of this study was to make a bibliometric and scientometric analysis of the articles on LA indexed in the Scopus database between 1989 and 2018. 118 publications were analyzed according to indicators of production, collaboration, impact and bibliometric maps. It was found that publications on LA have progressively increased, that the collaboration of authors was low, that an influential journal in the area was Library Review, and that the authors with the greater impact were Onwuegbuzie and Jiao. Also, keywords on user training and four research fronts were highlighted: measurement instruments, results, theoretical frameworks and types of analysis units. These results suggest that LA is a fruitful research topic.

Keywords: library anxiety; Bibliometrics; Scientometrics; information user; Scopus; VoSviewer; bibliographic coupling; co-citation.

Copyright: (c) 2021 CSIC. Este es un artículo de acceso abierto distribuido bajo los términos de la licencia de uso y distribución Creative Commons Reconocimiento 4.0 Internacional (CC BY 4.0). 


\section{INTRODUCCIÓN}

\subsection{Ansiedad y ansiedad bibliotecaria}

De forma general, y desde una perspectiva psicológica, la ansiedad se define como un estado emocional de "agitación e inquietud desagradable, caracterizado por la anticipación del peligro [...] y la sensación de catástrofe inminente" (Sierra y otros, 2003). Según Browne (2018), la ansiedad constituye un mecanismo biológico común y normal de las personas para enfrentar situaciones amenazantes. De forma más específica, la ansiedad ha sido estudiada en dos dimensiones: "como respuesta emocional y como rasgo de la personalidad" (García y Cano, 2014), denominadas ansiedad de estado y ansiedad de rasgo, respectivamente (Leal y otros, 2017). La ansiedad de rasgo es la característica estable de la personalidad de reaccionar de forma ansiosa, con independencia de la situación o circunstancia. Por el contrario, la ansiedad de estado es una reacción emocional transitoria vinculada a una situación específica (Arcas y Cano, 1999).

De acuerdo con ello, la ansiedad bibliotecaria ( $A B$ en adelante) se puede entender como el estado emocional de incertidumbre e incomodidad que experimentan los usuarios al momento de tener contacto con la biblioteca para requerir información. Mellon (1986) usó por primera vez el término y lo definió como "el sentimiento de temor" que experimentan los usuarios y "que les impide permanecer en la biblioteca", sin sacarle el máximo provecho para su formación. En este sentido, como indica Ramírez (2015), la AB es una manifestación de la ansiedad de estado. Es decir, es una manifestación transitoria en el usuario que se presenta en el contexto particular de la biblioteca y sus servicios. Onwuegbuzie y otros (2004) agregan que la $A B$ es un "estado negativo" que se da en un continuo, donde los usuarios "que experimentan los niveles más altos [de $A B$ ] tienen un mayor riesgo de exhibir conductas de evasión" (p. 55).

Considerando lo anterior, el estudio de la $A B$ se inscribe dentro del paradigma cognitivo en bibliotecología y ciencias de la información, donde se considera "variables afectivas, emocionales y cognitivas" (Tamayo-Rueda y Meneses-Placeres, 2018), como la actitud, las valoraciones, el comportamiento, la experiencia cognitiva del usuario, sus motivaciones y expectativas (Tamayo-Rueda y Meneses-Placeres, 2018; Izquierdo, 1999).

\subsection{Investigación sobre la ansiedad bibliote- caria}

Diversos estudios han buscado medir la $A B$ para determinar los factores que hacen que se manifieste. En general, dichos estudios siguen el enfoque cuantitativo y se centran en estudiar las percepciones de estudiantes universitarios sobre la biblioteca. Un ejemplo representativo es el trabajo de Ramírez (2015), quien agrega que es posible estudiar la $A B$ en un contexto latinoamericano. Para medir la $A B$ se han elaborado escalas psicométricas, como la escala de Bostick (Onwuegbuzie y otros, 2004), la escala multidimensional MLAS (Van Kampen, 2004) y la escala AQAK (Anwar y otros, 2012). Carlile (2007) hace una revisión de los trabajos sobre el tema publicados entre 1986 y 2006 y los clasifica según el enfoque de estudio, el tipo de artículo, la metodología aplicada y el tipo de escala empleada para medir el fenómeno. La autora encuentra que si bien la escala de Bostick es aceptada como confiable, los estudios no muestran ser concluyentes. Asimismo, encuentra que los autores más prolíficos son Jiao y Onwuegbuzie. Respecto a esto, Cleveland (2004), en su revisión de los principales trabajos de Jiao y Onwuegbuzie, concluye que ambos autores son claves para comprender la importancia de la $A B$ como un fenómeno real que debe ser estudiado en el campo bibliotecológico.

\subsection{Bibliometría y cienciometría}

La bibliometría es "la aplicación de los métodos matemáticos y estadísticos a la cuantificación de libros y otros medios de comunicación" (Pritchard, 1969). Es decir, es el conjunto de métodos cuantitativos útiles para describir y medir la literatura académica (Roemer y Borchardt, 2015); por ejemplo, para medir la cantidad de publicaciones y las citas que estas reciben (Cortés, 2007). Respecto a lo último, el análisis bibliométrico se basa en la idea de que existe un vínculo fuerte y directo entre las citas y el contenido de los artículos citados, "lo que significa que el análisis de citas puede ser el principal instrumento para evaluar el impacto de las publicaciones científicas" (Abramo y otros, 2019).

Por otro lado, la cienciometría es "el estudio de los aspectos cuantitativos de la ciencia y la tecnología vistos como un proceso de comunicación" (Mingers y Leydesdorff, 2015). Incluye dentro de sus principales temas la cuantificación de las investigaciones y su impacto, las citaciones, el mapeo de las áreas científicas y el uso de indicadores para las políticas y gestión de la investigación. Por esta razón, existe un solapamiento entre bibliometría y cienciometría, ya que ambas incluyen aspectos cuantitativos de la ciencia como proceso de comunicación (Björneborn y Ingwersen, 2004).

En bibliometría y cienciometría se usan indicadores para describir, evaluar y hacer seguimiento a las publicaciones (Riggio, 2017). Tradicionalmente se clasifican en indicadores de producción, de impacto 
Tabla I. Tipos de indicadores bibliométricos y cienciométricos

\begin{tabular}{|l|l|l|}
\hline Indicador & Descripción & Ejemplo \\
\hline Producción & $\begin{array}{l}\text { Cuantifica las publicaciones de autores, } \\
\text { instituciones o países, para determinar el } \\
\text { dinamismo del campo de investigación. }\end{array}$ & $\begin{array}{l}\text { Año, idioma, número y tipo del documento, } \\
\text { ciudad de afiliación, número de instituciones, } \\
\text { temática, índice de productividad, etc. }\end{array}$ \\
\hline Colaboración & $\begin{array}{l}\text { Describe y diagnostica las relaciones de } \\
\text { colaboración entre autores, instituciones o } \\
\text { países. }\end{array}$ & $\begin{array}{l}\text { Índice de colaboración, grado de colaboración, } \\
\text { coeficiente de colaboración, etc. }\end{array}$ \\
\hline Bmpacto & $\begin{array}{l}\text { Evalúa la relevancia de las publicaciones en } \\
\text { función del número de citas recibidas, para } \\
\text { determinar su influencia a corto plazo. }\end{array}$ & $\begin{array}{l}\text { Cuartiles y factor de impacto JCR (Journal } \\
\text { Citation Reports), cuartiles e índice de impacto } \\
\text { SJR (SCImago Journal Rank), número de citas, } \\
\text { h-Index, etc. }\end{array}$ \\
\hline Basado en mapas & $\begin{array}{l}\text { Identifica especialidades e interdisciplinariedad } \\
\text { en la investigación. Mapea los ejes de la } \\
\text { ciencia. }\end{array}$ & $\begin{array}{l}\text { Mapas de emparejamiento bibliográfico, } \\
\text { cocitación, coautoría y coocurrencia de } \\
\text { términos en palabras clave, títulos y } \\
\text { resúmenes. }\end{array}$ \\
\hline
\end{tabular}

Fuente: Riggio (2017), Peralta y otros (2015), y Yang y Ding (2012).

y de colaboración (Peralta y otros, 2015). Además de estos, se usan indicadores bibliométricos basados en mapas (Yan y Ding, 2012) (Tabla I).

Respecto a los indicadores de producción, el índice de productividad (IP en adelante) permite categorizar a los autores y se calcula con el logaritmo del número de publicaciones: si el IP $\geq 1$ el autor es un gran productor, si $0<$ IP $<1$ es un productor intermedio, si el IP $=0$ es un productor transitorio (Mohanty y otros, 2018). En el caso de los indicadores de colaboración, el índice de colaboración mide el peso promedio del número de autores que firman un documento; su valor mínimo es 1 , sin tener un valor máximo determinado (Lawani, 1981). El grado de colaboración señala la proporción de documentos escritos en coautoría múltiple; su valor oscila entre 0 y 1 , donde los valores próximos a 1 indican que existe mayor frecuencia de documentos con, por lo menos, dos autores (Subramanyam, 1983; Salas y otros, 2018). Por último, el coeficiente de colaboración refleja al mismo tiempo el número y la proporción de autores por documento; su valor oscila entre 0 y 1 , que tiende a 0 cuando predominan los trabajos de un solo autor (Ajiferuke y otros, 1988).

Sobre los indicadores de impacto, el número total de citas es un indicador básico que mide en enteros las veces que un ítem es citado (Waltman, 2016). Así, cuantas más citas tenga una publicación, mayor será su impacto. Al ser acumulativo, este indicador tiende a incrementarse con el tiempo, a diferencia de otros indicadores, como el promedio de citas recibidas, que no es acumulativo sino "independiente del número" (Waltman, 2016). En general, se considera que la citas y la citación "reflejan, con importantes limitaciones, aspectos relacionados al impacto y a la relevancia científica" (Aksnes y otros, 2019,).
Finalmente, dentro de los indicadores basados en mapas, el análisis de emparejamiento bibliográfico (AEB en adelante) permite relacionar "documentos que referencian [o citan] un mismo grupo de documentos" (Boyack y Klavans, 2010). Es decir, el AEB vincula dos ítems $A$ y $B$ que hacen referencia en su bibliografía a un tercer ítem $C$. Cuando ello sucede, se dice que $A$ y $B$ están emparejados bibliográficamente por $\mathrm{C}$. Así, cuanto mayor es el número de referencias comunes de dos publicaciones, "significa que ambas pertenecen a la misma área [de investigación]" (Habib y Afzal, 2017). Por otro lado, contrario al AEB, el análisis de cocitación ( $A C$ en adelante) permite relacionar dos publicaciones que son citadas juntas en las referencias de una tercera publicación (Marshakova-Shaikevich, 1973; Small, 1973). De acuerdo con MacCain (1991), el AC de revistas permite "destacar la relación entre temas, especializaciones de investigación, [así como] la organización de la literatura académica en varios niveles de generalidad" (p. 295). En el caso de dos o más autores, existe cocitación si un tercero los cita en sus referencias (van Eck y Waltman, 2014). En este sentido, cuando "dos autores son citados de manera frecuente en las mismas publicaciones, su relación es fuerte" (Perianes-Rodríguez y otros, 2016).

\subsection{Objetivos de la investigación}

Se hizo una búsqueda en Scopus, Web of Science y Google Académico y no se encontraron trabajos métricos que midan las publicaciones sobre $A B$. Considerando que el tema resulta de interés por estudiar la subjetividad del usuario y que tener un panorama métrico del mismo sería útil para los investigadores interesados, el objetivo principal de este trabajo es hacer un análisis bibliométrico y cienciométrico de los artículos sobre $A B$ indizados 
en la base de datos Scopus. Los objetivos específicos son los siguientes: (1) analizar la producción según publicación quinquenal, tipo de publicación, países, idioma, áreas de investigación e índice de productividad; (2) determinar la colaboración de autores mediante el índice de colaboración, el grado de colaboración y el coeficiente de colaboración; (3) determinar el impacto y las agrupaciones de fuentes y autores mediante el análisis de citas, de emparejamiento bibliográfico y de cocitación; y (4) describir las tendencias de investigación mediante redes de coocurrencia de palabras clave y de términos de los resúmenes.

\section{MÉTODO}

En general, se siguió una metodología de cinco fases: (1) recopilación de datos, (2) definición de unidades de análisis, (3) definición de unidades de medida, (4) reducción de dimensionalidad y distribución de visualizaciones y (5) análisis e interpretación de la visualización (Vargas-Quesada y de Moya-Anegón, 2007). A continuación se describe las cuatro primeras fases. La última se desarrolla a lo largo del apartado Resultados.

En primer lugar, para buscar y recuperar los datos objeto de análisis se usó Scopus, un recurso confiable que abarca en gran medida las áreas de Ciencias Sociales, Humanidades y Artes (Elsevier, 2019). La búsqueda se hizo en noviembre de 2019 y se usó el término "library anxiety" para encontrar coincidencias en los títulos, resúmenes y palabras clave de los documentos publicados entre 1960 y 2018. El primer resultado fue de 129 ítems publicados entre 1989 y 2018. Dicho resultado se delimitó según tipo de documento (solo artículos originales y artículos de revisión) y según tipo de fuentes (solo revistas). La cadena de búsqueda final fue: TITLE-ABS-KEY ("library anxiety") AND PUBYEAR < 2019 AND (LIMIT-TO (DOCTYPE, "ar") OR LIMIT-TO (DOCTYPE, "re")) AND (LIMIT-TO (SRCTYPE, "j")), con la que se obtuvo 119 artículos, de los cuales se descartó uno por no tratar sobre $A B$, sino sobre la ansiedad en general. Los 118 artículos restantes se exportaron en un archivo .CSV según las siguientes opciones en Scopus: información de citas, información bibliográfica, resumen y palabras clave, y otra información.

En segundo lugar, se definieron cinco unidades de análisis: documentos, fuentes, autores, palabras clave y términos en los resúmenes. En tercer lugar, se establecieron seis unidades de medida: producción, colaboración, citas, emparejamiento bibliográfico, cocitación y tendencia de investigación. En cuarto lugar, para analizar dichas unidades se usaron los programas Excel 2016, Publish or
Perish y VOSviewer v1.6.13. Los dos primeros sirvieron para realizar las tablas y gráficos de los indicadores de producción (según año, tipo, idioma, país, área de investigación e índice de productividad de autores) y la colaboración de autores (según índice, grado y coeficiente de colaboración). VOSviewer (Van Eck y Waltman, 2019) se usó para analizar las citas y crear los mapas de emparejamiento bibliográfico y de cocitación (según fuentes y autores), además de los mapas de tendencia de investigación (según coocurrencia de palabras clave y de términos en los resúmenes). Para crear la mayoría de estos mapas se usó el método fractional counting, el cual asigna el mismo peso a cada acción y es recomendado para este tipo de análisis (Perianes-Rodríguez y otros, 2016; van Eck y Waltman, 2014; Martorell y otros, 2019).

\section{RESULTADOS}

\subsection{Indicadores de producción}

La producción quinquenal sobre $A B$ en el periodo 1989-2018, según tipo de documentos (artículos originales y artículos de revisión), indicó la tendencia de crecimiento de publicaciones hasta el quinquenio 2014-2018 (Figura 1). Este quinquenio fue el más productivo con 42 artículos. Cabe destacar que desde 1996 se ha publicado al menos un documento por año. Respecto al tipo de documento, el artículo original fue el predominante con 109 ítems, frente al artículo de revisión con solo 9 ítems, el cual empezó a publicarse a mitad de los quinquenios indicados (2004-2008).

Figura 1. Artículos sobre ansiedad bibliotecaria indizados en Scopus según quinquenio

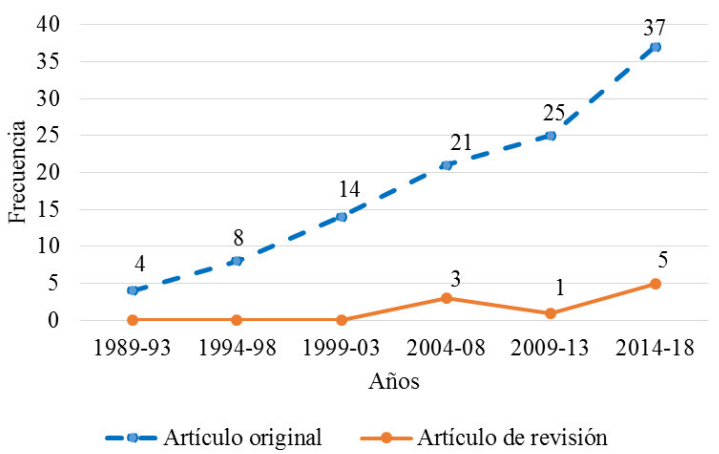

Respecto a la producción por país, la mayoría de documentos tuvo afiliación de Estados Unidos $(57 \%)$, seguido por Malasia $(8 \%)$ y Pakistán (6\%). Menos representados estuvieron Australia, Canadá, Georgia, Jamaica, Jordania, Irán, Israel, 
Kuwait, Sudán, Reino Unido ( $2 \%$ cada uno) y Bangladesh, China, Alemania, Indonesia, Japón, México, Polonia ( $1 \%$ cada uno). Un $3 \%$ no tuvo afiliación de país. Asimismo, la mayoría de documentos se escribió en inglés ( $98 \%$ ), y en menor proporción en español y japonés ( $1 \%$ cada uno).

Respecto a las áreas temáticas, se encontró que los 118 artículos estudiados se distribuyen en 9 áreas: Social Sciences (77\%), Computer Science (15\%), Arts and Humanities (2\%), Decision Sciences (2\%), Business, Management and Accounting $(1 \%)$, Medicine $(1 \%)$, Mathematics $(1 \%)$, y Psychology (1\%). Cabe resaltar que algunos de estos artículos están descritos en Scopus en más de un área de temática. También que las dos áreas predominantes, Social Sciences y Computer Science, acumularon el $92 \%$ de publicaciones, y que las áreas Arts and Humanities y Psychology estuvieron poco representadas.

Por otro lado, se encontró un total de 150 autores. La gran mayoría (87\%) tiene solo una publicación, mientras que el resto (13\%) tiene dos o más publicaciones. Se encontró que Onwuegbuzie, A. J. (22 artículos) y Jiao, Q. G. (19 artículos) son grandes productores, con un IP de 1.3 cada uno. Karim, N. H. A. (8 artículos) y Anwar, M. A. (6 artículos) son productores intermedios con un IP de 0.9 y 0.8 , respectivamente. Otros nueve autores intermedios tuvieron poca actividad (IP entre 0.6 y 0.3). Lo llamativo del campo de la $A B$ es que el resto de autores ( $87 \%$ ) son autores transitorios por tener una sola publicación. Es decir, la gran mayoría no suele seguir publicando sobre el tema después del primer trabajo.

\section{2. Índice, grado y coeficiente de colaboración}

Respecto a la colaboración de autores, el 57\% de los trabajos se escribieron en colaboración, frente al $43 \%$ que tuvo un solo autor (Tabla II). No obstante, la mayoría de colaboración se dio solamente entre dos autores (36\%). Cabe destacar que el quinquenio con mayor colaboración fue el periodo 2014-2018.
Figura 2. Índice, grado y coeficiente de colaboración de autores

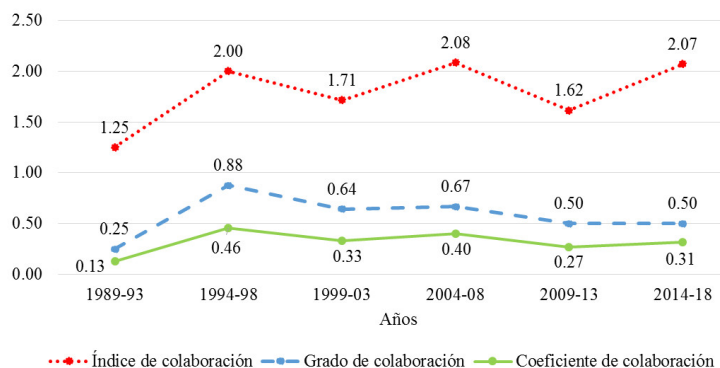

La tendencia de colaboración según el índice, el grado y el coeficiente de colaboración (IC, GC y CC, respectivamente), para cada quinquenio, indicó un bajo IC de autores, que osciló entre uno y dos en los últimos años (Figura 2). Asimismo, el GC con mayor proporción de coautoría ocurrió en el quinquenio 1994-1998 (0.88), en los inicios de la investigación sobre $A B$. No obstante, la tendencia fue que en los últimos años dicha proporción baje, siendo el período 2014-2018 el que presentó igualdad entre autores con una publicación y autores con al menos dos publicaciones. Por su parte, el CC tuvo un comportamiento similar al GC, con la diferencia que presentó valores menores. Es decir, en general la colaboración en el campo de la $A B$ fue baja.

\subsection{Análisis de citas, emparejamiento biblio- gráfico y cocitación de fuentes y autores}

\section{Fuentes}

Se encontró un total de 42 revistas que publicaron sobre AB. Entre las 10 revistas más citadas destacaron Library and Information Science Research con 534 citas, seguida de Library Review con 222 citas y College and Research Libraries con 204 citas (Tabla III). Es decir, estas fueron las revistas con mayor impacto en términos de citas recibidas.

Tabla II. Distribución de publicaciones según periodo y número de autores

\begin{tabular}{|c|c|c|c|c|c|c|c|}
\hline & \multicolumn{6}{|c|}{ Periodo } & \multirow{2}{*}{ Total } \\
\hline & $89-93$ & 94-98 & 99-03 & 04-08 & $09-13$ & $14-18$ & \\
\hline \multicolumn{8}{|c|}{ Número de autores } \\
\hline 1 & 3 & 1 & 5 & 8 & 13 & 21 & $51(43 \%)$ \\
\hline 2 & 1 & 6 & 8 & 7 & 11 & 9 & $42(36 \%)$ \\
\hline$\geq 3$ & 0 & 1 & 1 & 9 & 2 & 12 & $26(21 \%)$ \\
\hline Total & 4 & 8 & 14 & 24 & 26 & 42 & 118 \\
\hline
\end{tabular}


Tabla III. Diez revistas más citadas

\begin{tabular}{|l|c|c|}
\hline Revista & Documentos & $\begin{array}{c}\text { Total } \\
\text { de } \\
\text { citas }\end{array}$ \\
\hline $\begin{array}{l}\text { Library and Information } \\
\text { Science Research }\end{array}$ & 9 & 534 \\
\hline Library Review & 12 & 222 \\
\hline $\begin{array}{l}\text { College and Research } \\
\text { Libraries }\end{array}$ & 8 & 204 \\
\hline Library Quarterly & 2 & 101 \\
\hline $\begin{array}{l}\text { Reference and User Services } \\
\text { Quarterly }\end{array}$ & 6 & 96 \\
\hline $\begin{array}{l}\text { Journal of Academic } \\
\text { Librarianship }\end{array}$ & 4 & 64 \\
\hline $\begin{array}{l}\text { Malaysian Journal of Library } \\
\text { and Information Science }\end{array}$ & 7 & 55 \\
\hline Reference Services Review & 4 & 53 \\
\hline $\begin{array}{l}\text { Australian Academic and } \\
\text { Research Libraries }\end{array}$ & 2 & 47 \\
\hline $\begin{array}{l}\text { Journal of American Society } \\
\text { for Information Science and } \\
\text { Technology }\end{array}$ & 2 & 46 \\
\hline
\end{tabular}

Para determinar las agrupaciones de las revistas, se hizo un análisis de emparejamiento biblio- gráfico (AEB) (Figura 3). Se encontró, dentro de las revistas identificadas, dos agrupaciones principales: una cuyo foco fue Library Review (con una fortaleza de enlace de 207.8) y otra cuyo foco fue Malaysian Journal of Library and Information Science (con una fortaleza de enlace de 138.63). Es decir, el AEB permitió determinar que alrededor de estas revistas se emparejan las fuentes que tienen similares referencias en común.

Para complementar este resultado, se hizo un análisis de cocitación (AC) de fuentes (Figura 4). Se encontraron tres revistas predominantes según cocitación: College \& Research Libraries (con una fortaleza de enlace de 172.2), Library and Information Science Research (con una fortaleza de enlace de 160.5) y Library Review (con una fortaleza de enlace de 119.2). Cabe destacar que dentro de estas fuentes, dos no son revistas: la tesis doctoral The development and validation of the Library Anxiety Scale (1993) y el libro Library anxiety: theory, research and application (2004), con fortaleza de enlace de 47 y 36 respectivamente. En dicha tesis se propuso la escala de Bostick de $A B$ y en el mencionado libro se presenta una síntesis de la investigación en $A B$ desde 1986 hasta 2004.

Figura 3. Mapa de densidad de emparejamiento bibliográfico de revistas

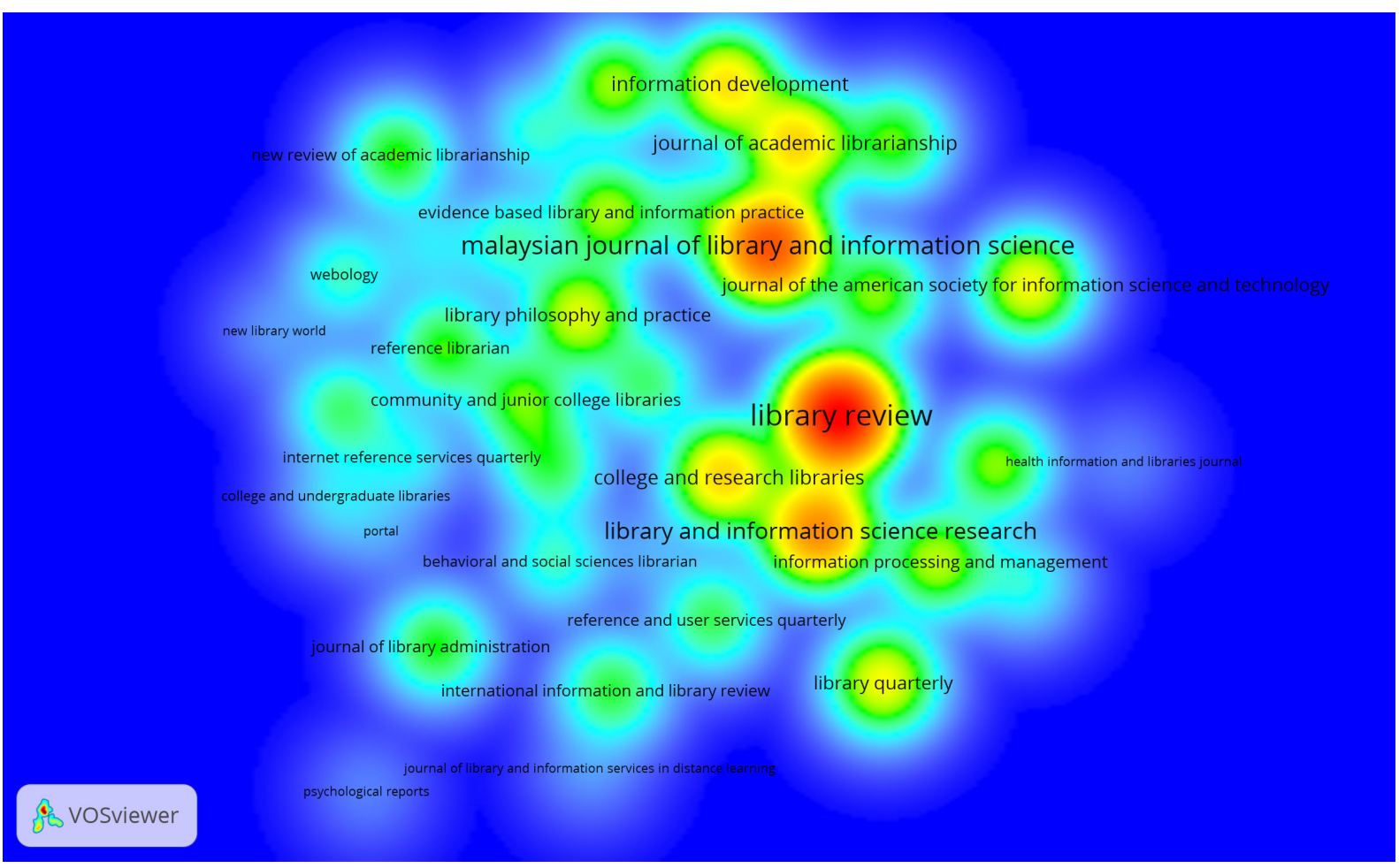

Nota: Se usaron revistas con un documento como mínimo. De las 42 revistas, solo 40 estuvieron conectadas entre sí. Método de conteo: fractional counting. Método de normalización: association strength. 
Figura 4. Mapa de cocitación de fuentes

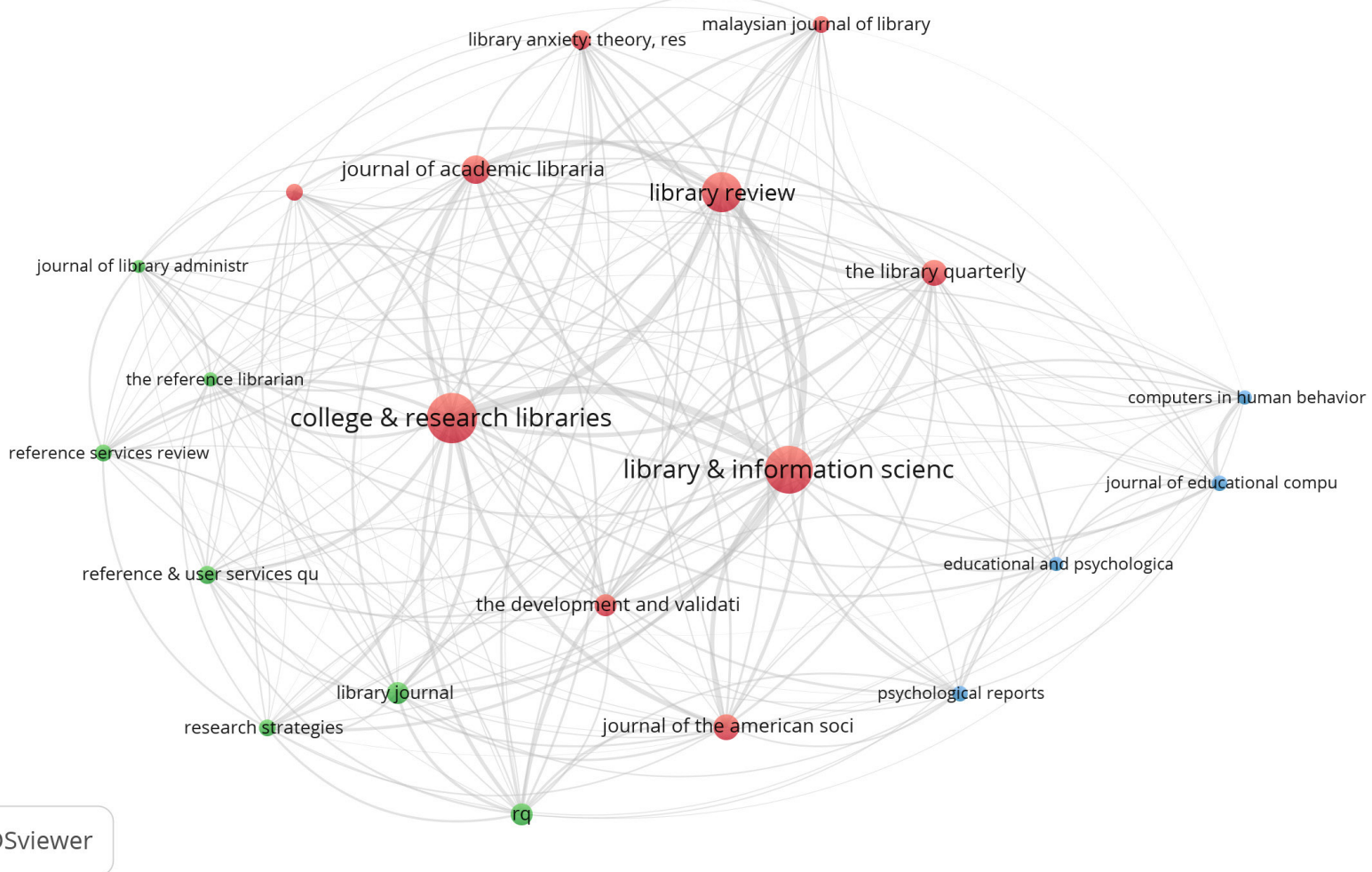

Nota: Se hizo un tesauro para estandarizar los nombres de las fuentes cocitadas en las referencias de los 118 documentos. Se usaron las fuentes que tuvieron 25 citas como mínimo. De las 1267 fuentes, solo 21 cumplieron dicho umbral. Método de conteo: fractional counting. Método de normalización: LinLog/modularity.

\section{Autores}

De los 150 autores identificados, Onwuegbuzie y Jiao, con 821 y 654 citas respectivamente, encabezaron la lista de los diez autores con mayor impacto en términos de citación (Tabla IV).

Tabla IV. Diez autores más citados

\begin{tabular}{|l|c|c|}
\hline Autores & Documentos & Total de citas \\
\hline Onwuegbuzie, A. J. & 22 & 821 \\
\hline Jiao, Q. G. & 19 & 654 \\
\hline Gross, M. & 1 & 123 \\
\hline Latham, D. & 1 & 123 \\
\hline Lichtenstein, A. A. & 1 & 99 \\
\hline Kwon, N. & 2 & 73 \\
\hline Anwar M. A. & 6 & 71 \\
\hline Al-kandari N. M. & 2 & 55 \\
\hline Al-qallaf C. L. & 2 & 55 \\
\hline Karim N. H. A. & 8 & 48 \\
\hline
\end{tabular}

Por medio del AEB (Figura 5), se encontraron dos grandes agrupaciones de autores: una cuyo foco lo formaron Onwuegbuzie y Jiao, con fortaleza de enlace de 935.4 y 824.61 , respectivamente; y otro cuyo foco lo formaron Karim y Anwar, con fortaleza de enlace de 212.77 y 197.75 , respectivamente. Básicamente, el primer grupo corresponde a autores con publicaciones clásicas sobre $A B$, mientras que el segundo grupo corresponde a los autores con publicaciones más actuales. En general, alrededor de dichos autores se emparejaron otros autores que tienen referencias en común.

Para complementar el análisis anterior, se indagaron sobre los autores más cocitados (Figura 6). De forma similar a los análisis anteriores, Onwuegbuzie y Jiao fueron autores relevantes, pues alcanzaron el mayor número de citas (572 y 437, respectivamente) y la mayor fuerza de enlace en cocitación (390 y 334 respectivamente). Los siguientes 3 autores relevantes fueron Mellon (con 137 citas y 142 de fuerza de enlace), Bostick (con 151 citas y 117 de fuerza de enlace) y Kuhlthau 
Figura 5. Mapa de densidad de emparejamiento bibliográfico de autores

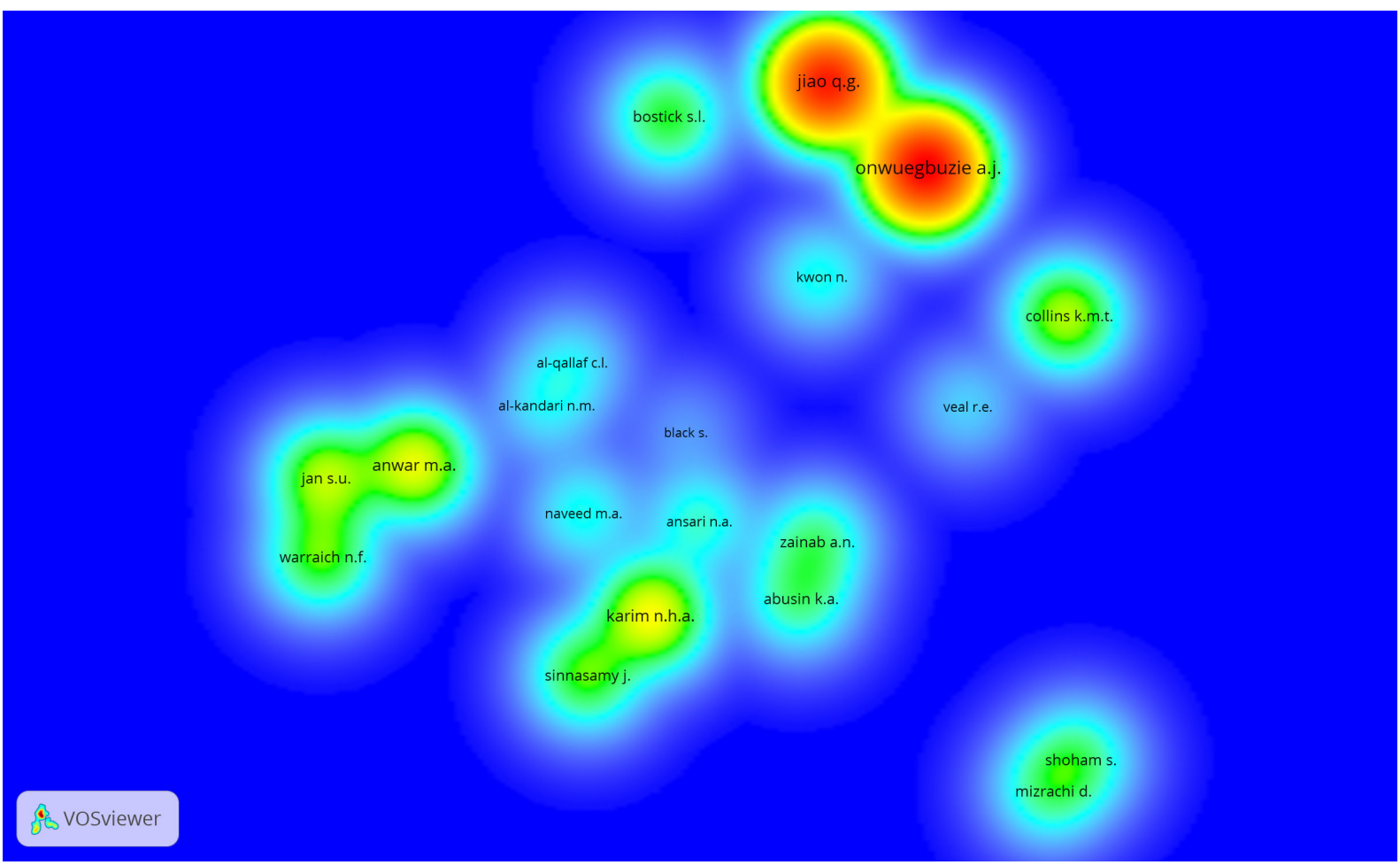

Nota: Se usaron autores con dos publicaciones como mínimo. De los 150 autores, 20 cumplieron dicho umbral. Método de conteo: fractional counting. Método de normalización: association strength.

Figura 6. Mapa de cocitación de autores

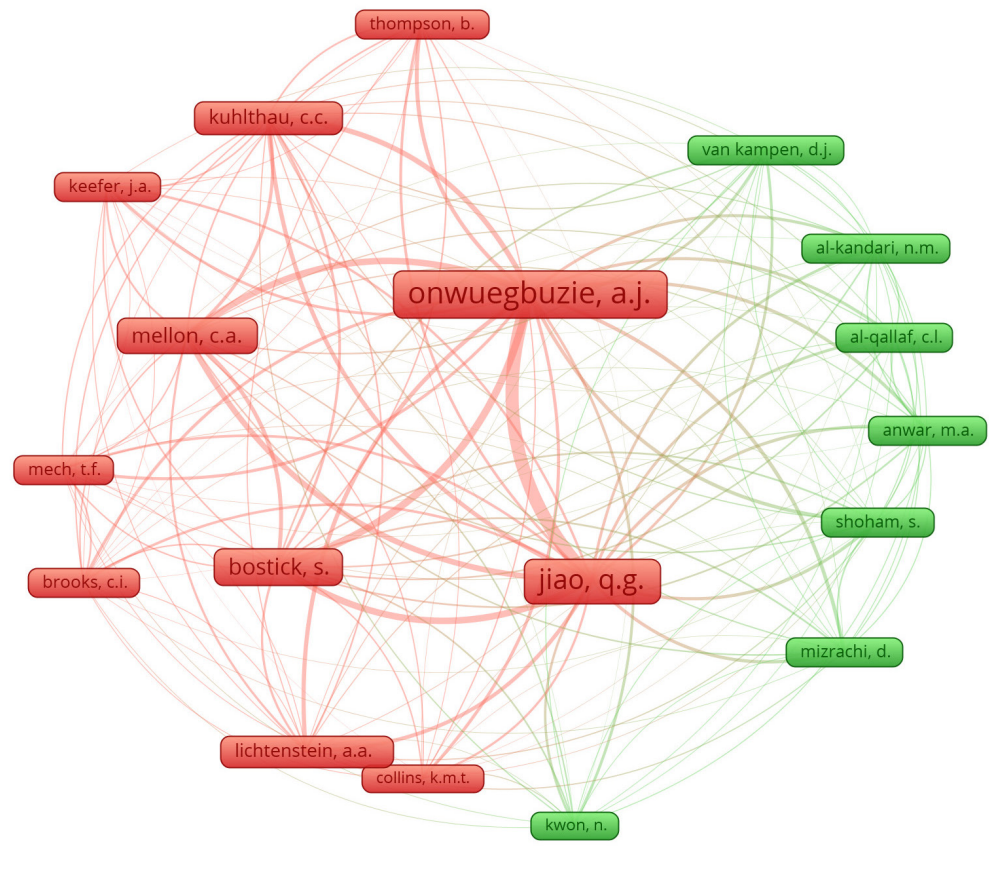

Nota: Se hizo un tesauro para estandarizar los nombres de los autores cocitados en las referencias de los 118 artículos. Se usaron los autores que tuvieran como mínimo 25 citas. De los 150 autores, solo 18 cumplieron dicho umbral. Método de conteo: fractional counting. Método de normalización: LinLog/modularity. 
(con 83 citas y 76 de fuerza de enlace), quienes hicieron aportes fundamentales a nivel teórico y metodológico con sus investigaciones. En ese sentido, estos autores fueron los más influyentes en términos de cocitación.

\subsection{Tendencias de investigación en el campo de ansiedad bibliotecaria}

De acuerdo al mapa de superposición de palabras claves de autores (Figura 7), algunos términos destacados en los 118 artículos fueron library anxiety, academic library, students, information literacy y library instruction (con 39, 23, 10, 8 y 8 de fuerza de enlace respectivamente). Respecto a la línea temporal, el término library anxiety sigue siendo vigente y su estudio ha sido relacionado tradicionalmente con los términos university libraries, students, USA y anxiety. En años posteriores, el estudio de la $A B$ se asoció con términos sobre la formación del usuario (information literacy y library instruction), y en años recientes con los términos emotional intelligence, information seeking anxiety, marketing y Pakistan.
Por último, el análisis de los términos extraídos de los resúmenes permitió visualizar 89 términos, los cuales se agruparon en cuatro frentes de investigación (Figura 8): (1) azul: términos relacionados a instrumentos de medición, como scale, instrument, sub scale y LAS (library anxiety scale). (2) Rojo: términos enfocado en marcos teóricos, como information, service, librarian, process. (3) Amarillo: términos asociados a los resultados, como implication, graduate student, role. (4) Verde: términos relacionados a tipos de unidades de análisis y sus características, como university student, library use, age, sex, native language.

\section{DISCUSIÓN Y CONCLUSIONES}

En primer lugar, la investigación en $A B$ ha tenido un crecimiento progresivo, aunque su presencia no es comparable al de otros tópicos de investigación en bibliotecología y ciencias de la información (Togia y Malliari, 2017). Sin embargo, esta es una oportunidad para estudiar el tema en regiones como Latinoamérica, que solo cuenta con el estudio de Ramírez (2015) (el 1\% de las publicaciones analizadas). Asi-

Figura 7. Mapa de superposición de palabras clave de autores

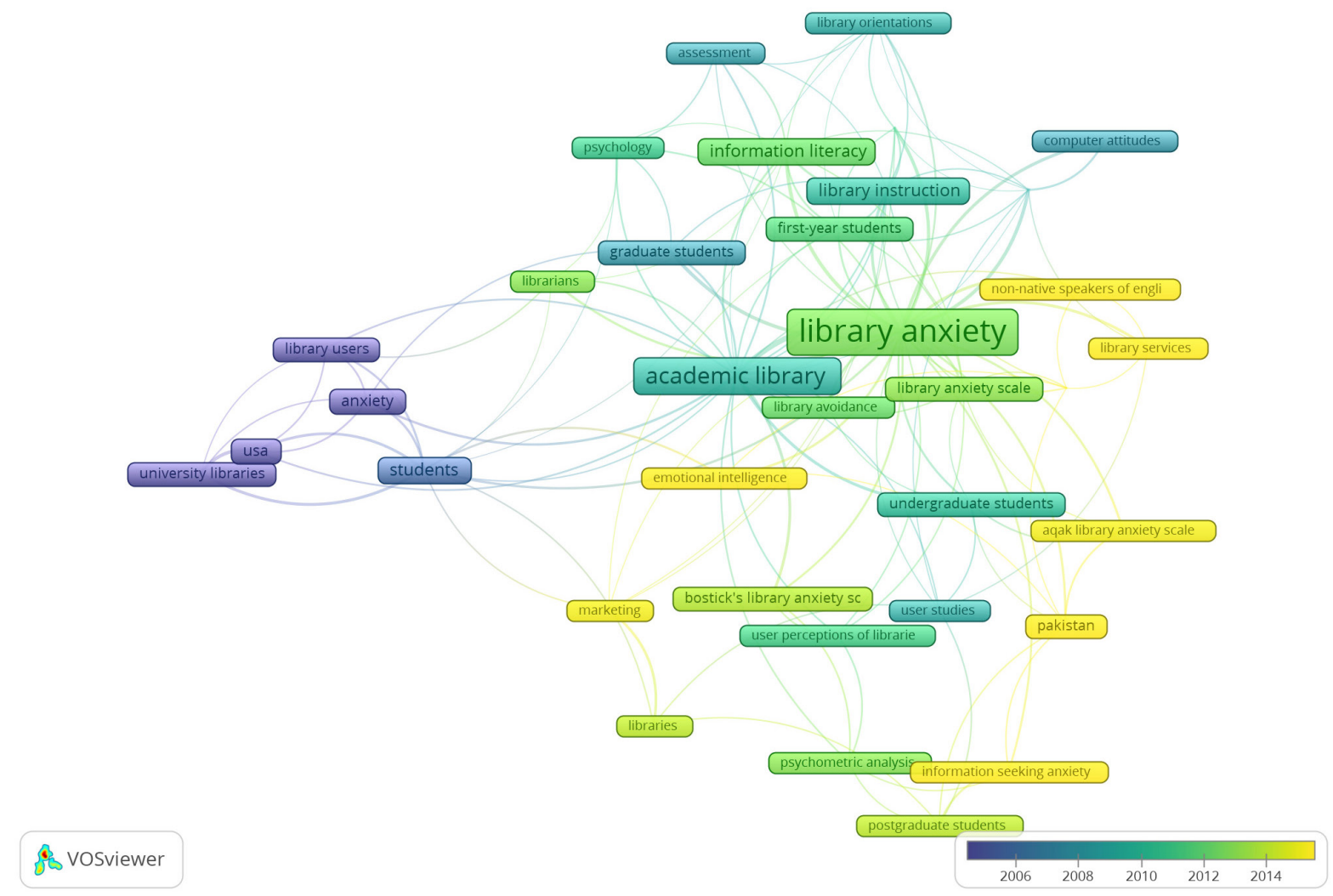

Nota: Se hizo un tesauro para estandarizar las palabras clave de autores. Se usaron solo las que tuvieran tres ocurrencias como mínimo. De las 205 palabras clave, solo 35 cumplieron dicho umbral. Método de conteo: fractional counting. Método de normalización: fractionalization. 
Figura 8. Mapa de red de términos en los resúmenes

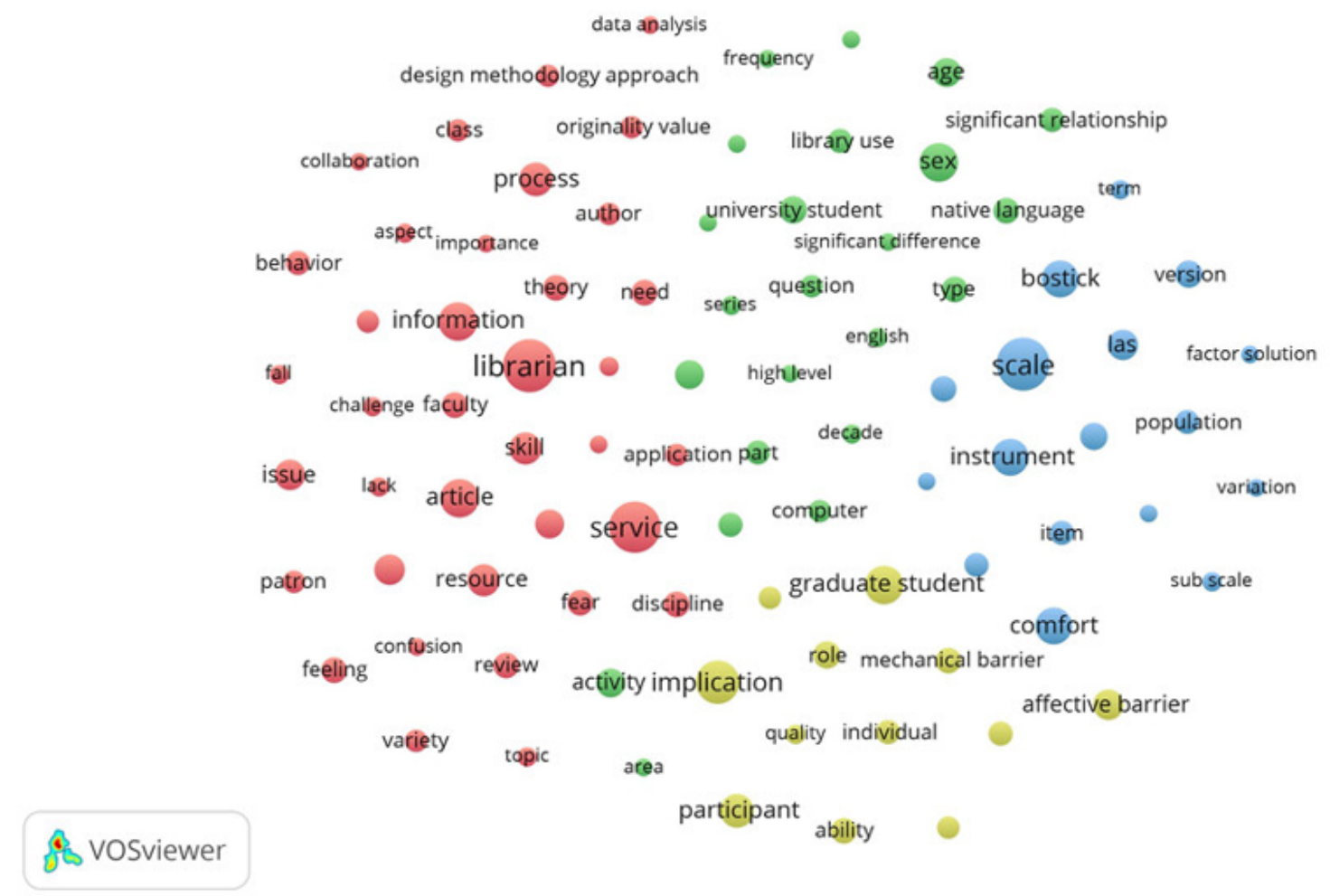

Nota: Se hizo un tesauro para estandarizar los términos de los resúmenes. Se usaron solo los que tuvieran cuatro ocurrencias como mínimo. Método de normalización: association strength.

mismo, se reafirmó lo señalado por Cleveland (2004) sobre la importancia de los autores Onwuegbuzie y Jiao, quienes además tienen trabajos en conjunto, aunque se evidenció que, en general, la tendencia de trabajos en colaboración fue baja. Esto resulta llamativo en una época de crecimiento de trabajos interdisciplinarios y de colaboración en diferentes áreas (Aldrich y Al-Turk, 2018; Hall y otros, 2018; Bu y otros, 2018). Quizá por ello las publicaciones estudiadas destacan en el área Social Science y no se observe mayor interdisciplinariedad. Solo se pueden destacar los trabajos desde el área Computer Science, donde se estudia la $A B$ asociada a la actitud y ansiedad hacia la tecnología (Jerabek y otros, 2001).

En segundo lugar, las revistas Library and Information Science Research y Library Review, fueron las de mayor impacto. Como complemento, las revistas Library Review y Malaysian Journal of Library and Information Science, así como los autores Onwuegbuzie, Jiao, Anwar y Karim generaron grandes agrupaciones de revistas y autores, respectivamente. Al respecto, según el portal Scimago, la revista Library and Information Science Research es la mejor posicionada en el cuartil 1 (Q1), seguida de Malaysian Journal of Library and Information Science en el cuartil 2 (Q2) y de Library Review ubicada en el cuartil 3 (Q3). De forma similar, según cocitaciones, otra revista relevante fue College \& Research Libraries, además de los autores Mellon, Bostick y Kuhlthau, quienes alcanzaron un alto número de citas y una alta fortaleza de cocitación. En resumen, el análisis descrito permitió tener un panorama de fuentes y autores relevantes, tanto con publicaciones clásicas como con investigaciones recientes.

En tercer lugar, la tendencia ha sido pasar de estudios sobre la naturaleza de la $A B$ hacia su relación con la formación del usuario (library instruction, information literacy). Otras relaciones interesantes se dan con los tópicos emotional intelligence, information seeking anxiety y marketing. Por otra parte, según el análisis de resúmenes, los estudios sobre $A B$ se han enfocado, básicamente, en estudiantes universitarios y no en usuarios de otras unidades de información. Asimismo, destacaron términos sobre elementos metodológicos de tipo cuantitativo, lo que sugiere una oportunidad para investigar la $A B$ desde enfoques cualitativos. Todo lo mencionado indica lo fructífero de investigar el tema, dependiendo de la perspectiva que se siga (Wildemuth, 2017). 
Finalmente, el estudio se basó solamente en artículos recuperados de Scopus y no en otro tipo de documento (capítulo de libro, editorial, conferencia y nota, que solo acumularon el $6 \%$ de las publicaciones sobre $A B$ ), por lo que se sugiere para futuros estudios incluir otros tipos de documentos y otras bases de datos. Del mismo modo, los mapas bibliométricos representaron solo parte de la actividad científica en el campo de la AB. No obstante, se buscó evidenciar la importancia de la bibliometría y cienciometría para describir temas de investigación en desarrollo como la $A B$, y suscitar investigaciones futuras al respecto dentro de la comunidad de bibliotecología y ciencias de la información, especialmente en Latinoamérica.

\section{REFERENCIAS}

Abramo, G., D’Angelo, C.; Reale, E. (2019). Peer review vs bibliometrics: which method better predicts the scholarly impact of publications? Scientometrics, 121(1), 537-554. https://doi.org/10.1007/s11192019-03184-y

Ajiferuke, I.; Burell, Q.; Tague, J. (1988). Collaborative coefficient: A single measure of the degree of collaboration in research. Scientometrics, 14(5-6), 421-433. https://doi.org/10.1007/BF02017100

Aksnes, D W.; Langfeldt, L.; Wouters, P. (2019). Citations, Citation Indicators, and Research Quality: An Overview of Basic Concepts and Theories. SAGE Open, 9(1), 1-17. https://doi. org/10.1177/2158244019829575

Aldrich, H.; Al-Turk, A. (2018). Crouching Authors, Hidden Pitfalls: Collaboration in Research. Studi Di Sociologia, 56(4), 341-328. https://doi. org/10.26350/000309_000041

Anwar, M.; Al-Qallaf, C.; Al-Kandari, N.; Al-Ansari, H. (2012). AQAK: A library anxiety scale for undergraduate students. Journal of Librarianship and Information Science, 44(1), 36-46. https://doi. org/10.1177/0961000611425568

Arcas, A.: Cano, A. (1999). Procesos cognitivos en el trastorno de ansiedad generalizada, según el paradigma del procesamiento de la información. Psicología.com: Revista Electrónica de Psicología, 3(1). Disponible en: http://psiqu.com/1-6380 [Fecha de consulta: 04/09/2019].

Björneborn, L. ; Ingwersen, P. (2004). Toward a basic framework for webometrics. Journal of the American Society for Information Science and Technology, 55(14), 1216-1227. https://doi.org/10.1002/asi.20077

Boyack, K.; Klavans, R. (2010). Co-Citation Analysis, Bibliographic Coupling, and Direct Citation: Which Citation Approach Represents the Research Front Most Accurately? Journal of the American Society for Information Science and Technology, 61(12), 23892404. https://doi.org/10.1002/asi.21419

Browne, D. (2018). Anxiety: Overview, symptoms, causes, and treatments. Disponible en: https://www. medicalnewstoday.com/articles/323454.php [Fecha de consulta: 04/09/2019]

\section{AGRADECIMIENTOS}

Se agradece al grupo de investigación CIGETMEN de la Facultad de Letras y Ciencias Humanas de la Universidad Nacional Mayor de San Marcos, y a la Dirección de Investigación de la Universidad Peruana de Ciencias Aplicadas.

\section{ACKNOWLEDGEMENTS}

Thanks to the research group CIGETMEN of the Facultad de Letras y Ciencias Humanas, Universidad Nacional Mayor de San Marcos, and to the Dirección de Investigación de la Universidad Peruana de Ciencias Aplicadas.

Bu, Y.; Ding, Y.; Liang, X.; Murray, D. S. (2018). Understanding persistent scientific collaboration. Journal of the Association for Information Science and Technology, 69(3), 438-448. https://doi.org/10.1002/asi.23966

Carlile, H. (2007). The implications of library anxiety for academic reference services: A review of literature. Australian Academic and Research Libraries, 38(2), 129-147. https://doi.org/10.1080/00048623.2007.10721282

Cleveland, A. (2004). Library anxiety: A decade of empirical research. Library Review, 53(3), 177-185. https:// doi.org/10.1108/00242530410526583

Cortés, D. (2007). Medir la producción científica de los investigadores universitarios: la bibliometría y sus límites. Revista de Educación Superior, 36(142), 4365. Disponible en: https://www.redalyc.org/articulo. oa?id=60414203 [Fecha de consulta: 11/09/2019].

Elsevier. (2019). ¿Qué es Scopus? [En línea]. Disponible en: https://service.elsevier.com/app/answers/detaiI/a_id/15534/supporthub/scopus/\#tips [Fecha de consulta: 02/10/2019].

García, Z.; Cano, A. (2014). Estandarización y validación del Inventario de Situaciones y Respuestas de Ansiedad (ISRA) en la población de República Dominicana. Summa Psicológica UST, 11(2), 81-99. Disponible en https://dialnet.unirioja.es/servlet/articulo?codigo $=4954000$ [Fecha de consulta: 21/09/2012]. https://doi.org/10.18774/448x.2014.11.130

Habib, R.; Afzal, M. (2017). Paper recommendation using citation proximity in bibliographic coupling. Turkish Journal of Electrical Engineering \& Computer Sciences, 25, 2708-2718. https://doi.org/10.3906/elk-1608-180

Hall, K. L.; Vogel, A. L.; Huang, G. C.; Serrano, K. J.; Rice, E. L.; Tsakraklides, S. P.; Fiore, S. M. (2018). The science of team science: A review of the empirical evidence and research gaps on collaboration in science. American Psychologist, 73(4), 532-548. https://doi. org/10.1037/amp0000319

Izquierdo, M. (1999). Una aproximación interdisciplinar al estudio del usuario de información: bases conceptuales y metodológicas. Investigación Bibliotecológica: Archivonomía, Bibliotecología e Información, 13(26), 112-134. https://doi.org/10.22201/ iibi.0187358xp.1999.26.3902 
Jerabek, J. A.; Meyer, L. S.; Kordinak, S. T. (2001). "Library anxiety" and "computer anxiety:" Measures, validity, and research implications. Library \& Information Science Research, 23(3), 277-289. https://doi. org/10.1016/s0740-8188(01)00083-4

Lawani, S. M. (1981). Bibliometrics: Its Theoretical Foundations, Methods and Applications. Libri, 31(4), 294315. https://doi.org/10.1515/libr.1981.31.1.294

Leal, P.; Goes, T.; da Silva, L.; Teixeira-Silva, F. (2017). Trait vs. state anxiety in different threatening situations. Trends Psychiatry Psychother, 39(3), 147-157. https://doi.org/10.1590/2237-6089-2016-0044

Marshakova-Shaikevich, I. (1973). System of documentation connections based on references (SCI). Nauchno Tekhnicheskaya Informatsiya Seriya, 2(6), 3-8. Disponible en: http://garfield.library.upenn.edu/marshakova/ marshakovanauchtechn1973.pdf [Fecha de consulta: 01/10/2019].

Martorell, O.; Socias, A.; Otero, L.; Mulet-Forteza, C. (2019). Thirty-fifth anniversary of the International Journal of Hospitality Management: A bibliometric overview. International Journal of Hospitality Management, 78, 89-101. https://doi.org/10.1016/j. ijhm.2018.10.013

McCain, K. W. (1991). Mapping economics through the journal literature: An experiment in journal cocitation analysis. Journal of the American Society for Information Science, 42(4), 290-296. https://doi. org/10.1002/(SICI)1097-4571(199105)42:4<290: :AI D-ASI5>3.0.CO;2-9

Mellon, C. A. (1986). Library Anxiety: A Grounded Theory and Its Development. College \& Research Libraries, 47(2), 160-165. https://doi.org/10.5860/crl.76.3.276

Mingers, J.; Leydesdorff, L. (2015). A review of theory and practice in scientometrics. European Journal of Operational Research, 246, 1-19. https://doi.org/10.1016/j. ejor.2015.04.002

Mohanty, B.; Sahoo, J.; Dash, N. (2018). Bibliometric Indicators for Assessing the Quality of Scholarly Communications: A Case Study on International Journal of Cooperative Information Systems. Library Philosophy and Practice (e-Journal). Disponible en: https://digitalcommons.unl.edu/libphilprac/2158 [Fecha de consulta: 01/10/2019].

Onwuegbuzie, J.; Jiao, Q.; Bostick, S. (2004). Library Anxiety: Theory, Research, and Applications. EE. UU: Scarecrow Press Inc. Disponible en: https://cutt.ly/ Wrezi5D [Fecha de consulta: 11/09/2019].

Peralta, M.; Frías, M.; Chaviano, O. (2015). Criterios, clasificaciones y tendencias de los indicadores bibliométricos en la evaluación de la ciencia. Revista Cubana de Información En Ciencias de La Salud, 26(3), 290309. Disponible en: http://dspace.uclv.edu.cu/handle/123456789/5101 [Fecha de consulta: 18/09/2019].

Perianes-Rodriguez, A.; Waltman, L.; van Eck, N. (2016). Constructing bibliometric networks: A comparison between full and fractional counting. Journal of Informetrics, 10(4), 1178-1195. https://doi.org/10.1016/j. joi.2016.10.006

Pritchard, A. (1969). Statistical bibliography or bibliometrics? Journal of Documentation, 25(4), 348-349. Disponible en: https://www.researchgate.net/publi- cation/236031787 Statistical Bibliography or Bibliometrics [Fecha de consulta: 02/09/2019].

Ramírez, N. (2015). Ansiedad bibliotecaria en estudiantes universitarios. Revista Interamericana de Bibliotecología, 38(3), 227-236. https://doi.org/10.17533/udea. rib.v38n3a07

Riggio, G. (2017). Indicadores bibliométricos de la actividad científica de la República Dominicana. [Tesis doctoral]. Madrid: Universidad Carlos III. Disponible en: http://eprints.rclis.org/31698/ [Fecha de consulta: 01/10/2019]

Roemer, R.; Borchardt, R. (2015). Meaningful Metrics: A 21st-Century Librarian's Guide to Bibliometrics, Altmetrics, and Research Impact. Chicago, Illinois: Association of College and Research Libraries. Disponible en: http:// www.ala.org/acrl/sites/ala.org.acrl/files/content/publications/booksanddigitalresources/digital/9780838987568_ metrics_OA.pdf [Fecha de consulta: 11/09/2019].

Salas, G.; Ravelo-Contreras, E. L.; Mejía S., Andrades R., Acuña E., Espinoza F.; Núñez, M.; Barboza-Palomino, M.; Ventura-León, J.; Caycho-Rodríguez, T.; Pérez-Acosta, A. M. (2018). Dos décadas de Acta Colombiana de Psicología: un análisis bibliométrico. Acta Colombiana de Psicología, 21(2), 13-38. https://doi. org/10.14718/acp.2018.21.2.2

Sierra, J. : Ortega, V.; Zubeidat, I. (2003). Ansiedad, angustia y estrés: tres conceptos a diferenciar. Revista Mal-Estar e Subjetividade, 3(1), 10-59. Disponible en: https:// biblat.unam.mx/es/revista/revista-mal-estar-e-subjetividade/articulo/ansiedad-angustia-y-estres-tres-conceptos-a-diferenciar [Fecha de consulta: 04/09/2019].

Small, H. (1973). Co-citation in the scientific literature: A new measure of the relationship between two documents. Journal of the American Society for Information Science, 24(4), 265-269. https://doi.org/10.1002/ asi. 4630240406

Subramanyam, K. (1983). Bibliometric studies of research collaboration: A review. Journal of Information Science, 6(1), 33-38. https://doi. org/10.1177/016555158300600105

Tamayo-Rueda, D.; Meneses-Placeres, G. (2018). Comportamiento informacional: revisión de teorías posibles para su estudio. E-Ciencias de La Información, 8(2), 2-19. https://doi.org/10.15517/ECI.V8I2.32441

Togia, A.; Malliari, A. (2017). Research Methods in Library and Information Science. En: Oflazoglu, S. (ed.). Qualitative Versus Quantitative Research, pp. 43-64. IntechOpen. doi:10.5772/intechopen.68749. https://doi. org/10.5772/intechopen.68749

Van Eck, N.; Waltman, L. (2014). Visualizing bibliometric networks. En Ding, Y.; Rousseau, R.; Wolfram, D. (Eds.), Measuring scholarly impact: Methods and practice. pp. 285-320. Springer. https://doi. org/10.1007/978-3-319-10377-8_13

Van Eck, N.; Waltman, L. (2019). VoSviewer Manual. Disponible en: https://www.vosviewer.com/documentation/Manual VOSviewer 1.6.10.pdf [Fecha de consulta: 25/09/2019].

Van Kampen, D. (2004). Development and Validation of the Multidimensional Library Anxiety Scale. College and Research Libraries, 65(1), 28-34. https://doi. org/10.5860/crl.65.1.28 
Vargas-Quesada, B.; de Moya-Anegón, F. (2007). Visualizing the structure of science. New York, NY: Springer. Disponible en: https://www.springer.com/gp/ book/9783540697275.

Waltman, L. (2016). A review of the literature on citation impact indicators. Journal of Informetrics, 10(2), 365-391. https://doi.org/10.1016/j.joi.2016.02.007

Wildemuth, B. (2017). Library Anxiety Impedes College Students' Library Use, but May Be Alleviated Through
Improved Bibliographic Instruction. Evidence Based $\mathrm{Li}$ brary and Information Practice, 12(4), 275. https:// doi.org/10.18438/b8k082

Yang, E.; Ding, Y. (2012). Scholarly Network Similarities: How Bibliographic Coupling Networks, Citation Networks, Cocitation Networks, Topical Networks, Coauthorship Networks, and Coword Networks Relate to Each Other. Journal of the American Society for Information Science and Technology, 63(7), 1313-1326. https:// doi.org/10.1002/asi.22680 